\title{
Fast simulation of showers in the H1 calorimeter
}

\author{
A. Glazov ${ }^{\mathrm{a}}$, N. Raičević ${ }^{\mathrm{b}, *}$, A. Zhokin ${ }^{\mathrm{c}}$ \\ a Deutsches Electronen-Synchrotron, DESY, Notkestrasse 85, D-22607 Hamburg, Germany \\ b University of Montenegro, Faculty of Science, Džorža Vašingtona BB, 20000 Podgorica, Montenegro \\ ${ }^{\mathrm{c}}$ Institute for Theoretical and Experimental Physics, B. Cheremushkinskaja 25, Moscow 117259, Russia
}

\section{A R T I C L E I N F O}

\section{Article history:}

Received 30 September 2009

Received in revised form 14 January 2010

Accepted 2 February 2010

Available online 6 February 2010

\section{Keywords:}

Shower library

Shower parametrisation

Fast simulation

SpaCal

\begin{abstract}
A B S T R A C T
A method for the fast simulation of particle showers in the H1 lead/scintillating-fiber calorimeter is presented. It is based on a shower library technique in which the detector response is simulated using a collection of stored showers for different particle types and topologies. The library is created using the GEANT programme. The fast simulation is compared to the data collected by the H1 experiment.
\end{abstract}

(c) 2010 Elsevier B.V. All rights reserved.

\section{Introduction}

Simulation of high energy physics experiments requires large computational resources. A significant fraction of CPU time is typically spent for the simulation of showers in calorimeters. Various techniques have been developed to accelerate the simulation of showers. These include parametrisation methods, e.g. GFLASH [1], shower libraries [2] and so-called frozen showers [3].

A "shower library" is a collection of stored GEANT [4] energies in the calorimeter for a given particle type, energy and location at the calorimeter surface. The structure of the shower library depends on the construction of the calorimeter and particle kinematics. The shower library provides description of the detector response approaching the quality of the GEANT simulation while the computing time is much reduced.

In the $\mathrm{H} 1$ detector [5] from the HERA accelerator in Hamburg, the shower library is implemented for the lead/scintillating-fiber calorimeter (so-called "Spaghetti calorimeter" - SpaCal) [6]. The $\mathrm{H} 1$ detector is dedicated to the study of the deep inelastic scattering process using colliding electron and proton beams of $25.5 \mathrm{GeV}$ and $920 \mathrm{GeV}$, respectively. The SpaCal calorimeter is comprised of electromagnetic and hadronic sections. The electromagnetic part of the SpaCal consists of 1192 cells with an active volume of $4.05 \times 4.05 \times 25 \mathrm{~cm}^{3}$ each. A transverse view of the calorimeter is given in Fig. 1. The cells are made of grooved lead plates and scintillating fibers with a diameter of $0.5 \mathrm{~mm}$. The scintillation

\footnotetext{
* Corresponding author.

E-mail addresses: glazov@mail.desy.de (A. Glazov), raicevic@mail.desy.de (N. Raičević), zhokin@mail.desy.de (A. Zhokin).
}

light of each cell is converted into an electric pulse using photomultiplier tubes. The active length of the electromagnetic SpaCal corresponds to 27.5 radiation lengths and about one hadronic interaction length. The Moliere radius of the SpaCal is $2.5 \mathrm{~cm}$. The angular coverage of the calorimeter, which is measured with respect to the proton beam direction, ${ }^{1}$ is $153^{\circ}<\theta<177.8^{\circ}$.

The electromagnetic energy resolution can be parametrised as $7.5 \% / \sqrt{E} \oplus 2 \%$. The spatial resolution of the calorimeter in the transverse plane is about $3.5 \mathrm{~mm}$. The amount of material from the interaction point to the calorimeter surface varies between 0.6 and 1.0 radiation length.

The hadronic part of the SpaCal is comprised of 136 cells of $12 \times 12 \times 25 \mathrm{~cm}^{3}$ providing one nuclear interaction length. The fibers are of the same type as in the electromagnetic section but have a larger diameter of $1 \mathrm{~mm}$.

The SpaCal is used for the reconstruction of electron and positron clusters obtained from $e^{ \pm} p$ scattering. A good description of cluster properties is important for the identification of the scattered lepton and rejection of hadronic background. This is of particular interest for the measurement of the proton structure function, $F_{L}$, which is performed in a high background kinematic domain. The first measurement of $F_{L}$ reported by the H1 Collaboration [7] was obtained using Monte Carlo simulation which was based on the shower library described in this paper.

This paper is organised as follows: in Section 2 a general description of the shower library is given; grid, interpolation, struc-

\footnotetext{
1 The $z$-axis of the right-handed coordinate system used by $\mathrm{H} 1$ is defined to lie along the direction of the incident proton beam and the origin to be at the nominal ep interaction vertex.
} 


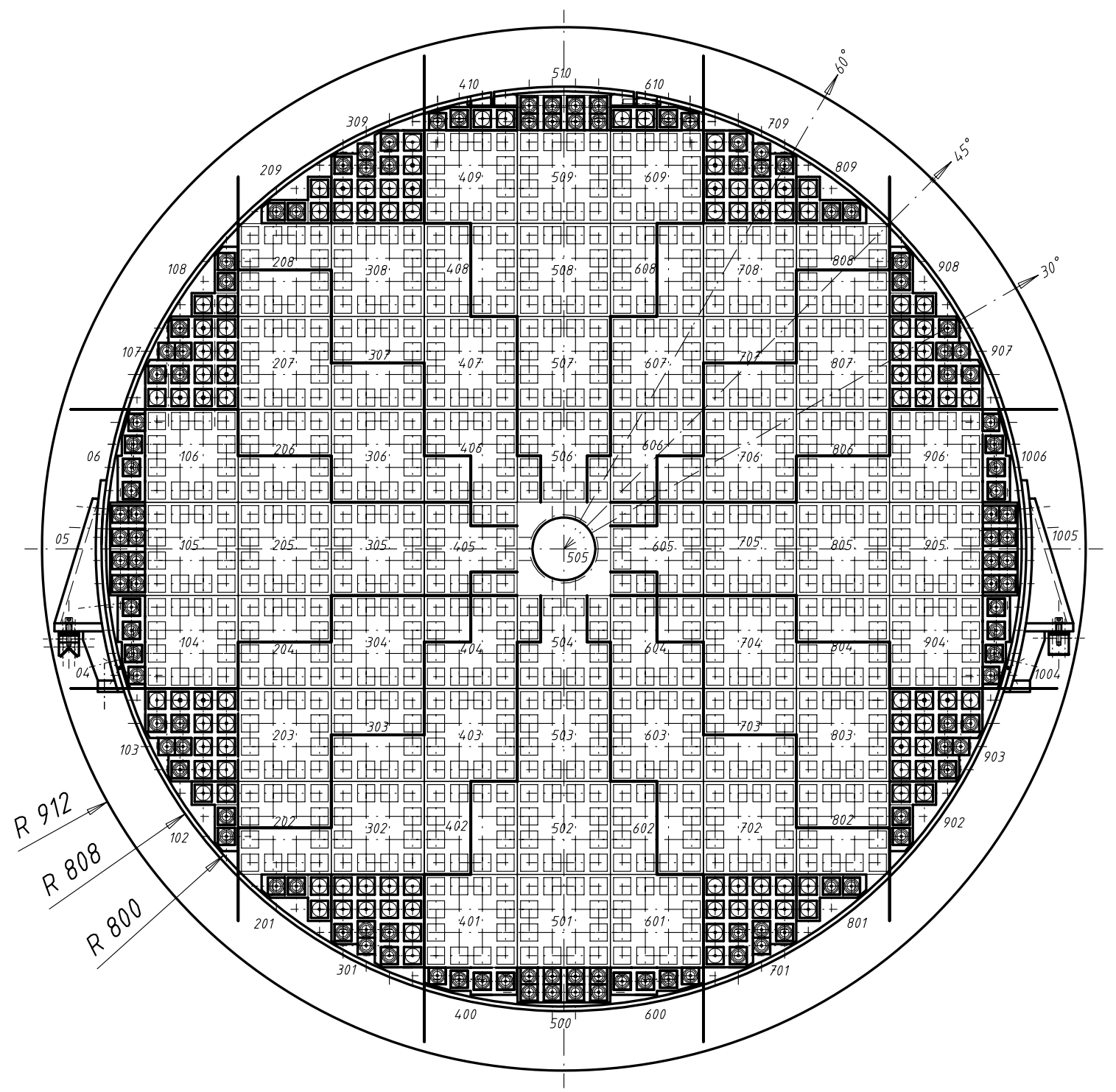

Fig. 1. Transverse view of the SpaCal. Small boxes indicate individual cells. They are combined in $4 \times 4$ groups into super-modules.

ture and packing are discussed; in Section 3 implementation of the shower library for the SpaCal is presented; in Section 4 the simulations based on shower library and shower parametrisation [1] techniques are compared to the $\mathrm{H} 1$ data.

\section{Shower library}

In the simulation using the shower library a generated particle is traced through the detector components up to the calorimeter surface. At this point, if the impact position of the particle is far enough from the calorimeter boundaries, such that the shower is expected to be fully contained in the calorimeter, the shower library is applied: instead of using the GEANT package to simulate the calorimeter response, a suitable pre-simulated shower is selected from the shower library. The selected shower is then corrected for a difference between the actual particle kinematics and the kinematics of the particle used for the shower creation. Corrected energy deposits are added to the calorimeter response.

The quality of the simulation based on the shower library therefore depends on how well it represents kinematics of simulated particles, how many showers are stored for each grid point and how large residual corrections are. Since the computer memory is limited, an additional important feature of the shower li- brary is the packing scheme. These issues are briefly discussed in the next paragraphs.

\subsection{Grid and interpolation}

The shower library grid is binned in the particle energy, $E$, in the particle position with respect to the calorimeter surface, $x$ and $y$, and in the particle direction angles, $\theta_{x}$ and $\theta_{y}$, with respect to the lepton beam direction which is perpendicular to the calorimeter surface.

The binning in energy should be fine enough to describe the energy resolution properly. Typically it is spaced uniformly in $\log E$. The grid should start from low energy, $\simeq 100 \mathrm{MeV}$, to describe showers starting before the calorimeter surface which contain many particles at low energy. The granularity of the position binning parallel to the calorimeter surface should be fine enough compared to the calorimeter spacial resolution. Symmetries of the calorimeter can be used to reduce the number of position bins. A reduction is possible for a large (vs shower size) calorimeter with translation symmetry. In this case it is sufficient to use position and angular binning inside the cell with the largest energy deposit ("hottest cell"). Further reduction is possible by mapping the hottest cell of the calorimeter to itself using $x-y$ rotations and mir- 


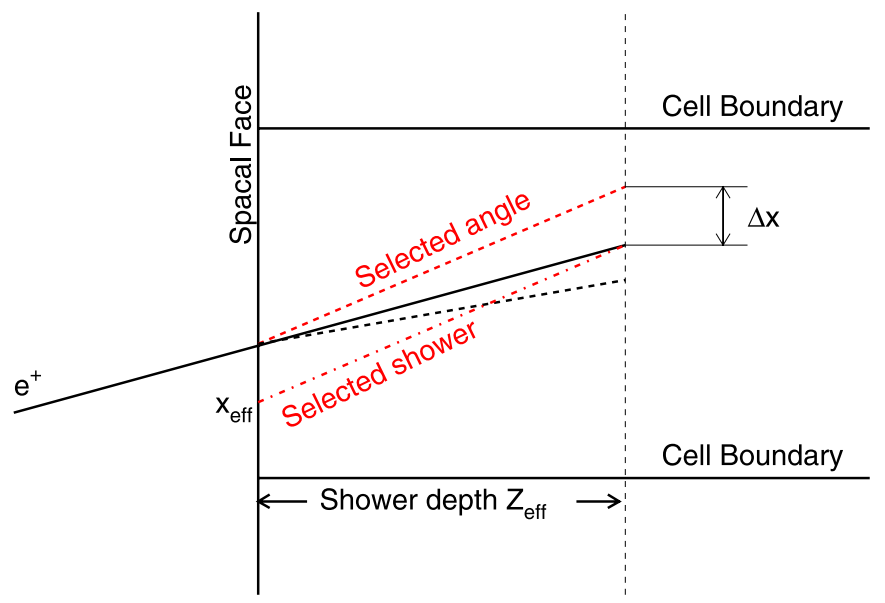

Fig. 2. Schematic representation of the angular correction algorithm, see text.

ror images. The granularity of the angular binning usually does not need to be too fine, if the angles are sufficiently small. A small angle $\theta_{x, y}$ leads to only a moderate (proportional to $\cos \theta_{x, y}$ ) increase of the transverse shower size which is usually not very important. A larger effect is introduced by an effective shift of the shower $x, y$-position. This effect can be compensated for using an effective shower depth as will be discussed later.

The shower library is created using the GEANT simulation for particles selected with energies, positions and angles as defined by the shower library grid. For a given grid point, typically several showers are stored.

For simulation using the shower library, the bin which best fits the particle kinematics is selected. Two different algorithms are used to determine the energy and position bins. The energy bin is selected using the following algorithm:

1. For a given particle energy, $E_{\text {gen }}$, find the nearest higher and lower energy grid points, $E_{h}$ and $E_{l}\left(E_{l}<E_{\text {gen }} \leqslant E_{h}\right)$.

2. With a probability proportional to the "distance" from a grid point to $E_{g e n}$, randomly select an $E_{h}$ or $E_{l}$ bin. The "distance" can be measured linearly or logarithmically. For the logarithmic measure, which is used for the SpaCal shower library, the probability to select the lower grid point is:

$$
P_{l}=\frac{\log \frac{E_{h}}{E_{g e n}}}{\log \frac{E_{h}}{E_{l}}} .
$$

3. For the selected bin ( $h$ or $l$ ), take the shower from the shower library and, before adding to the calorimeter response, re-scale the energies of deposits as $E_{g e n} / E_{l, h}$ ( $E_{l, h}$ stands for $E_{l}$ or $E_{h}$ ).

This procedure adequately reproduces the variation of the energy resolution as a function of energy correctly up to first order in $\log E_{h} / E_{l}$.

The particle position bin is determined using the following algorithm, illustrated in Fig. 2:

1. Effective shower depth $\left(z_{\text {eff }}\right)$ for electromagnetic showers is estimated. It depends logarithmically on the particle energy.

2. The shower library angular bins $\theta_{x, y}$ are selected using the linear interpolation algorithm in the same manner as for the energy bin.

3. The particle trajectory is extrapolated up to $z_{\text {eff }}$ along the original particle direction and then extrapolated back to the calorimeter surface using the selected $\theta_{x, y}$-directions. This defines the effective impact point, $x_{\text {eff }}, y_{\text {eff }}$.
4. $x_{e f f}, y_{\text {eff }}$ are used to select the $x, y$-position using linear interpolation.

\subsection{Structure and packing}

To obtain a good description of the calorimeter response and avoid statistical biases, the shower library should be sufficiently large. In order to avoid relatively slow disk access it is preferred to have the showers needed for all grid points buffered in the computer physical memory. In the case of multidimensional binning (in $x, y, \theta_{x}, \theta_{y}, E$ and particle type) and fine calorimeter granularity (hundreds of energy deposits per shower), the total memory requirement can be rather high.

A usual strategy is to use buffers of packed showers. One buffer covers one complete shower library which covers all grid points and stores at least one shower per grid point. Several buffers are stored in a disk file. The showers from one buffer reside in memory, and are reused several times; a new buffer is read from the disk file after the repetition count exceeds some fixed value.

Electromagnetic showers are typically small in size and they can be stored using energy deposits in the cells in a fixed box around the hottest cell. To pack shower information effectively, it is usual to store the total energy of the shower as an uncompressed real number; the energy deposits are stored in terms of a bit packed fraction of the total energy. The fraction of energy of the hottest cell should be stored with the highest precision (e.g. with 16 bits) while for peripheral cells, just few bits can be sufficient.

For broad, asymmetric hadronic showers, many cells may contain no energy deposit. In this case, it is more efficient to store information only from the cells which contain energy. All these options are introduced in the H1 shower library implementation.

\section{Shower library for SpaCal}

The parameters of the shower library for the SpaCal are given in Table 1 . The shower library for electromagnetic particles contains 12 bins in energy ranging logarithmically from $0.1 \mathrm{GeV}$ up to $32 \mathrm{GeV}, 10$ bins in $x$ and 10 bins in $y$ which cover the front face of the hottest cell, 8 bins in $\theta_{x}$ and 8 bins in $\theta_{y}$ ranging from $-24.8^{\circ}$ to $24.8^{\circ}$ and two bins for particle type, for electron/positron ${ }^{2}$ and photon. The total number of grid bins is

\section{$12 \cdot 10 \cdot 10 \cdot 8 \cdot 8 \cdot 2=153600$}

which corresponds to one buffer of the shower library.

The shower library for hadrons contains 10 bins in energy from $0.1 \mathrm{GeV}$ up to $20 \mathrm{GeV}, 5$ bins in $x$ and 5 bins in $y$ which cover the front face of the hottest cell, 4 bins in $\theta_{x}$ and 4 bins in $\theta_{y}$ from

Table 1

Parameters of the SpaCal shower library for electromagnetic and hadronic particles.

\begin{tabular}{lll}
\hline Parameter & $\begin{array}{l}\text { Shower library for } \\
\text { electromagnetic particles }\end{array}$ & $\begin{array}{l}\text { Shower library } \\
\text { for hadrons }\end{array}$ \\
\hline Energy binning & $12,0.1-32 \mathrm{GeV}$ & $10,0.1-20 \mathrm{GeV}$ \\
Position binning & $10 \times 10$ in $x, y$ & $5 \times 5$ in $x, y$ \\
Angular binning & $8 \times 8$ in $\theta_{x}, \theta_{y}$ & $4 \times 4$ in $\theta_{x}, \theta_{y}$ \\
Particle type & 2 & 9 \\
binning & $e^{ \pm}, \gamma$ & $\pi^{ \pm}, K^{ \pm}, K_{L}, n, \bar{n}, p, \bar{p}$ \\
Showers per buffer & 153600 & 36000 \\
Shower size & $7 \times 7(\mathrm{ELM})+5 \times 5$ (HAD) cells & up to 1328 cells \\
\hline
\end{tabular}

\footnotetext{
2 A detailed study of the showers produced by positrons and electrons shows that they are virtually identical; therefore no additional binning distinguishing electrons and positrons is introduced.
} 
$-12.1^{\circ}$ to $12.1^{\circ}$ and 9 bins in particle type. The total number of grid bins is

$10 \cdot 5 \cdot 5 \cdot 4 \cdot 4 \cdot 9=36000$

which corresponds to one buffer of the shower library.

The shower library for electromagnetic particles is built of showers containing the energy response of 49 cells from the electromagnetic section and 25 cells from the hadronic section. The shower library for hadrons is built of showers containing the energy responses from all cells in the electromagnetic and hadronic sections (1328) with non-zero energy. The packing of the energy information reduces the size for one buffer to about $8 \mathrm{MB}$.

Due to interactions in the material in front of the calorimeter, a single electron from the interaction point creates on average 30 particles that impact the calorimeter. The repetition count for reading a new buffer is set to the total number of showers in the buffer, 153600, thus one buffer is used to simulate about 5000 electrons. There are in total 7 buffers used for the SpaCal simulation. Since the shower developement up to the SpaCal is random and the resulting SpaCal shower is build from many showers from the shower library, the number of showers in the library is sufficient for the $\mathrm{H} 1$ analyses which study up to $\sim 10^{7}$ scattered electrons.

\section{Results and discussion}

The main quantity used for particle identification in the SpaCal is the transverse size of the shower parametrised by a cluster radius. The determination of the cluster radius starts with the calculation of the cluster centre-of-gravity which is defined as a weighted average over the positions of the cluster cell centres:

$X_{\text {cluster }}=\sum_{i} x_{i} w_{i}, \quad Y_{\text {cluster }}=\sum_{i} y_{i} w_{i}$.

Here $x_{i}, y_{i}$ are the $x$ and $y$ coordinates of the $i$-th cell centre and $w_{i}$ defines the weight of cell $i$. Two definitions are used in $\mathrm{H} 1$ to calculate $w_{i}$, based on square root and logarithmic energy weighting:

$w_{i, \mathrm{sqrt}}=\frac{\sqrt{E_{i}}}{\sum_{j} \sqrt{E_{j}}}$,

$w_{i, \log }=\frac{\max \left(0, w_{\text {cut }}+\log \left(E_{i} / E_{\text {cluster }}\right)\right)}{\sum_{j} \max \left(0, w_{\text {cut }}+\log \left(E_{j} / E_{\text {cluster }}\right)\right)}$.

Here $E_{i}$ corresponds to the energy reconstructed in the cell $i$, and $w_{\text {cut }}$ defines the logarithmic cut-off parameter for the SpaCal taken to be 4.85 .

The cluster radius is then calculated as a weighted sum over the distances, $R_{i}$, between the centre of each cell, $i$, and the cluster centre-of-gravity. Corresponding to the two definitions of weighting, two cluster radius calculations are employed at H1. These are, so-called,

$E C R A=\sum_{i} R_{i} w_{i, \text { sqrt }}$,

and the logarithmic cluster radius,

$R_{\log }=\sqrt{\sum_{i}\left(R_{i} w_{i, \log }\right)^{2}}$.

SpaCal clusters corresponding to the scattered positrons, from $e^{+} p$ interactions, are compared between the $\mathrm{H} 1$ data and simulation. For this comparison data collected by the H1 Collaboration in the year 2000 are used. For these data, positrons with energy

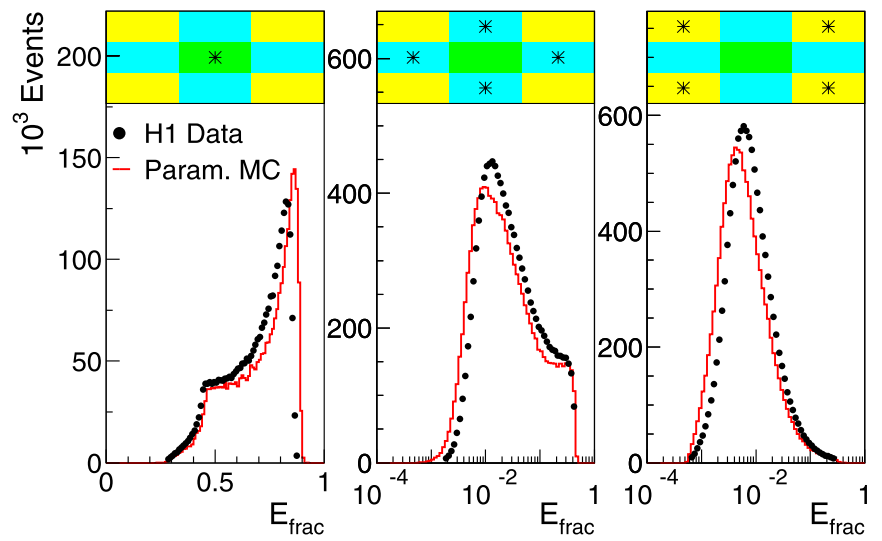

Fig. 3. Comparison of $\mathrm{H} 1$ data (dots) with Monte Carlo simulation using the GFLASH based shower parametrisation (histogram) for cluster energy fraction contained in the hottest cell (left), in all cells closest to the hottest cells (middle) and in all cells diagonal with respect to the hottest one (right). Cells for which the energy fraction is calculated are marked with a star in the $3 \times 3$ box above corresponding distributions.

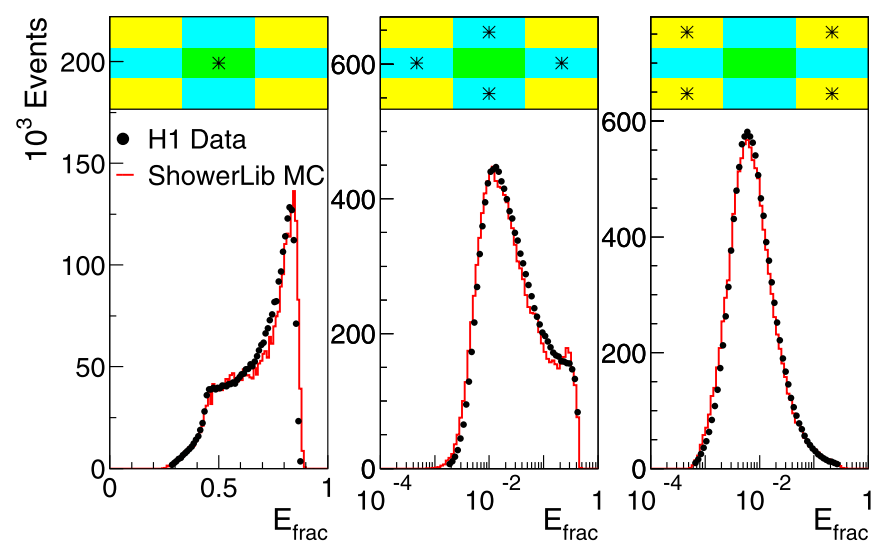

Fig. 4. Comparison of $\mathrm{H} 1$ data (dots) with Monte Carlo simulation using the shower library (histogram) for cluster energy fraction contained in the hottest cell (left), in all cells closest to the hottest cells (middle) and in all cells diagonal with respect to the hottest one (right). Cells for which the energy fraction is calculated are marked with a star in the $3 \times 3$ box above corresponding distributions.

of $27.5 \mathrm{GeV}$ collided with protons with energy of $920 \mathrm{GeV}$. The simulation of $e^{+} p$ collisions uses the DJANGO event generator [8].

A pure sample of scattered positron clusters is obtained by requiring the energy of the cluster to exceed $15 \mathrm{GeV}$; this avoids hadronic background contamination. The selected cluster is required to be in the region of the SpaCal far from the inner and outer acceptance edges.

Figs. 3 and 4 show comparisons between the $\mathrm{H} 1$ data and Monte Carlo simulation using the GFLASH based shower parametrisation [1] and between data and Monte Carlo simulation using the shower library. The comparisons are made for the cluster energy fraction contained in the hottest cell, in all cells closest to the hottest cell and in all cells diagonal with respect to the hottest cell. The simulation using the shower library provides fair description of the shower profile.

Similar comparisons are shown in Figs. 5 and 6 for the $R_{\log }$ and ECRA estimators. Using the shower library, a good description of the cluster radius is achieved.

Both simulation methods, using the shower library and the shower parametrisation, are about ten times faster than the full GEANT simulation for the scattered positron energies in the tested range. 

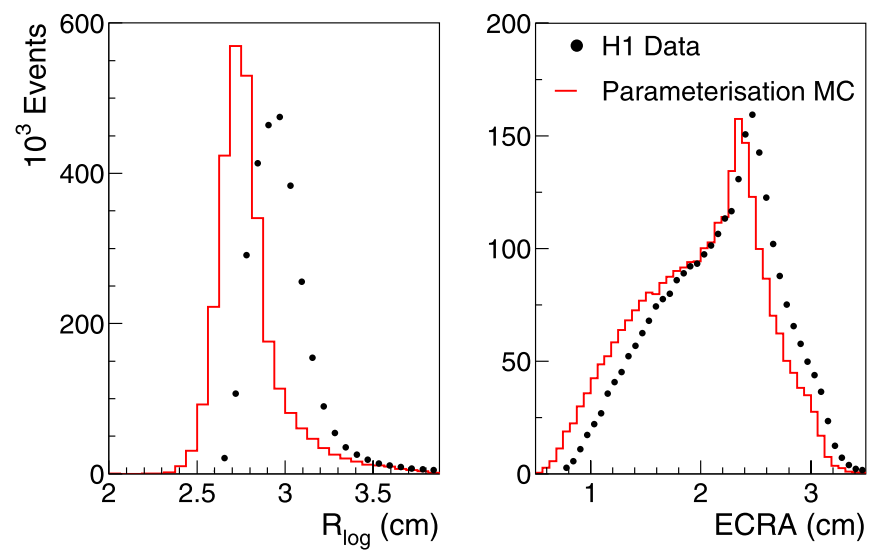

Fig. 5. Comparison of $\mathrm{H} 1$ data (dots) with simulation using the GFLASH based shower parametrisation (histogram) for $R_{\log }$ (left) and ECRA (right).
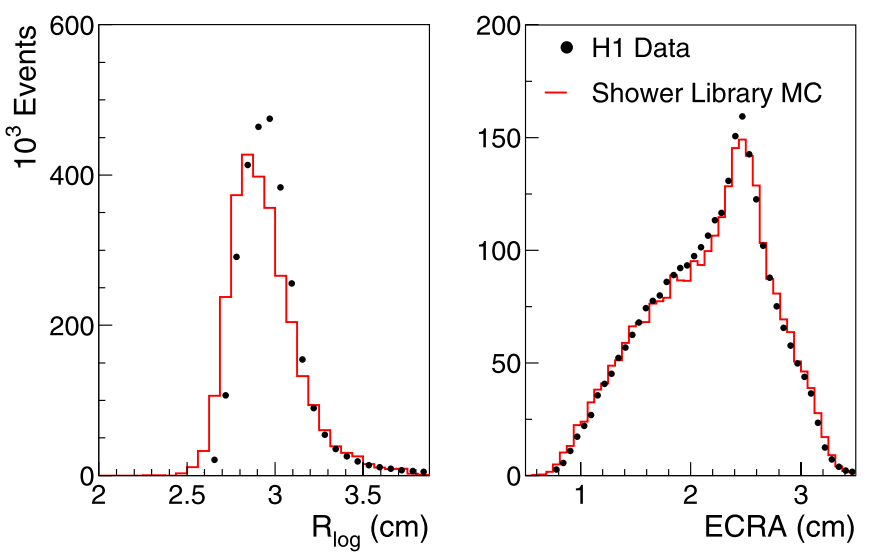

Fig. 6. Comparison of H1 data (dots) with Monte Carlo simulation using the shower library (histogram) for $R_{\log }$ (left) and ECRA (right).

\section{Conclusion}

An implementation of the shower library for the simulation of the $\mathrm{H} 1$ detector is presented. The software to generate, store and read back the shower library information is described. The shower library is created for the lead/scintillating-fibre calorimeter SpaCal.

Monte Carlo simulation using the shower library provides good description of the cluster shapes observed in data. The CPU time is reduced compared to the full GEANT simulation by about a factor of ten.

\section{Acknowledgements}

We would like to thank J. Ferencei, E. Turner Worchester, and D. Pitzl for reading this manuscript and fruitful discussions. We are grateful to the H1 Collaboration for providing the data for benchmarking of the simulation.

\section{References}

[1] G. Grindhammer, M. Rudowicz, S. Peters, Nucl. Instr. Methods A 290 (1990) 469;

G. Grindhammer, S. Peters, hep-ex/0001020

[2] E. Turner Worcester, Ph.D. thesis, University of Chicago, Chicago, 2007.

[3] E. Barberio, et al., J. Phys. Conf. Ser. 160 (2009) 012082.

[4] R. Brun, et al. CERN GEANT.3, DD/EE/84-1, 1987; Application Software Group, GEANT Detector Description, Simulation Tool, CERN Program Library Long Writeup W5013, October, 1994.

[5] I. Abt, et al., H1 Collaboration, Nucl. Instr. Methods A 386 (1997) 310; I. Abt, et al., H1 Collaboration, Nucl. Instr. Methods A 386 (1997) 348

[6] R.-D. Appuhn, et al., H1 SpaCal Group, Nucl. Instr. Methods A 386 (1997) 397.

[7] F.D. Aaron, et al., H1 Collaboration, Phys. Lett. B 665 (2008) 139.

[8] G.A. Schuler, H. Spiesberger, in: W. Buchmuller, G. Ingelman (Eds.), Proc. Workshop on HERA Physics, vol. 3, DESY, Hamburg, 1992, p. 1419; A. Kwiatkowski, H. Spiesberger, H.-J. Mohring, Comput. Phys. Comm. 69 (1992) 155. 